\title{
Research development in tumor therapy: role of iron-related nanoparticles
}

\author{
Dai Peipei ${ }^{1}$ \\ ${ }^{1}$ School of Pharmaceutical Science \& Technology, Tianjin University, Tianjin 300072, China.
}

\begin{abstract}
As an essential nutrient element for life, iron's metabolic balance in body tissues is crucial to sustaining normal physiological functions, and it is inextricably related to tumors. Nanotechnology is gaining much attention around the world for cancer treatment. Considering the critical role of iron metabolism, nanocarriers' toxicity and biocompatibility, novel nanomaterials based on the biochemical activity of iron and the regulatory proteins of iron homeostasis-metabolism show broad application prospects in the field of tumor diagnosis and treatment. In this review, the role of iron-related nanocarriers for tumor therapy, such as iron oxide nanoparticles, Fe-based metal-organic frameworks, ferritin, and transferrin, was reviewed, aiming to help people better understand their tremendous potential in tumor therapy.
\end{abstract}

\section{Introduction}

Cancer is one of the leading causes of death worldwide, posing a serious threat to global public health and harming countless people's quality of life and health. Conventional cancer treatments cover surgery, radiotherapy, chemotherapy, and so on. Nevertheless, these methods are a "double-edged sword" with numerous side effects $[1,2]$. Therefore, more and more scientists are devoted to exploring new cancer treatment strategies, especially the concept based on drug delivery, which aims to develop drug-delivery vectors to achieve controlled drug release, thereby lessening side effects and advancing the pertinence and effectiveness of treatment.

Nanotechnology has been popularly adopted in the biomedical field and foreseen to present a significant driver for innovative diagnostics and treatments for cancer. Adjustable size, tailorable framework, and other fascinating physical and chemical properties of nanoparticles (NPs) allow them to be designed as nanoplatforms with specific applications, such as drug delivery and controlled drug release, passive or active targeting to tumors through enhanced permeability and retention (EPR) effects and surface modification, and both diagnostic and therapeutic properties [3]. This makes nanomaterials one of the most promising cancer treatments to offer personalized cancer therapy with capabilities and efficacy that surpass traditional technologies and methods.

Iron is intimately associated with human life and found almost omnipresent, which makes up about 5 percent of the earth's crust at $7.55 \times 10^{17}$ tons, ranking fourth on the element abundance list [4]. Iron has good ductility, electrical and thermal conductivity, and excellent ferromagnetism. At the same time, human cells also have a v ast iron pool. Amongst more than a dozen trace elements essential to the human body, iron ranks first both in value and in quantity. Cells have established various sophisticated mechanisms to control their iron homeostasis strictly, including ferritin (Ft) [5], transferrin (TF) [6], and various iron-regulating proteins [7-9].

NPs applied in cancer diagnosis and treatment mainly fall into polymeric NPs, polymeric micelles, dendrimers, liposomes, metal NPs, crystalline NPs, carbon-based NPs and natural biological NPs [10]. Based on the crucial role of iron and its regulatory molecules in the human body, the relationship between iron and tumors, as well as other considerations such as physicochemical properties, cost, production difficulty, inherent biocompatibility, and biodegradation, many current studies have focused on iron-based NP carriers and ironrelated natural biomolecular carriers. In this paper, from the perspective of iron physicochemical properties and iron metabolism, the potential clinical applications of iron-related nanocarriers like iron oxide nanoparticles (SPIONs), Fe-based metal-organic frameworks (MOFs), $\mathrm{TF}$, and $\mathrm{Ft}$, are principally introduced for tumor diagnosis and treatment. This review is anticipated to contribute to understanding the fabulous potential of iron-related NPs in cancer therapy.

\section{Iron in human body}

\subsection{Regulation of iron metabolism}

Iron in the human body can be classified into two categories: functional iron and storage iron. This mineral

\footnotetext{
* Corresponding author: peipeidai@tju.edu.cn
} 
is largely present in the form of ferric hemoglobin and ferry myoglobin. Iron is also a cofactor of many essential enzymes. These enzymes are closely related to complex biochemical reactions such as biological oxidation, tissue respiration, energy metabolism, DNA synthesis, and body immunity $[11,12]$. Given the influence of iron, it is particularly necessary to regulate iron metabolism and maintain iron homeostasis in the human body. Management of iron metabolism requires complex and fine coordination of various proteins, and the more important ones are Ft [5], TF [6], hepcidin [8], and various iron ion regulatory proteins [7].

As an iron-storing protein, Ft can be detected in almost all body tissues, notably the liver. The primary duty of this spherical cage structure is to store and regulate iron ions [5]. The transport of iron in the body cannot leave the main iron transport protein in plasmaTF [6]. Iron uptake by cells exists mainly on the interaction between TF and transferrin receptor 1 (TFR1) [13]. Iron is bound to $\mathrm{TF}$ and undergoes a conformational change to form the TRF-Fe3+ complex, which binds to the TFR1 on the cell surface and enters the cell through pinocytosis. After iron release, the TFTFR1 complex returns to the cell surface, and the TF detaches from the receptor and re-enters the bloodstream, and the process repeats [14].

A series of iron ion regulatory proteins are also required to maintain iron homeostasis. Among them, the currently known core role is hepcidin, a negative regulator of iron metabolism [8]. It regulates cellular iron's outflow by binding the only cellular iron efflux protein-ferroportin (FPN1) [7, 15, 16]. Besides, iron sensor proteins IRP2, an iron regulatory protein in the cytoplasm, can sense the drop of iron concentration in the cell and bind to iron response elements on mRNAs such as Ft and TFR to command the translation of these proteins and regulate the intracellular iron concentration [9]. There is a sophisticated regulation system for iron homeostasis in the human body, and too many factors that directly or indirectly regulate iron homeostasis are involved. Some complex molecular mechanisms are still under study.

\subsection{Relationship between iron and tumor}

Lack or overload of iron can have adverse health effects. In addition to causing iron deficiency anemia [17], iron deficiency is harmful in many ways. Because of the lack of iron, the human body of many iron-containing enzyme functions will decline, so that the substance and energy metabolism are affected $[11,12,18]$.

The most direct reason for cell damage caused by iron overload is that excessive free iron can catalyze the generation of free radicals, promote oxidative stress, induce lipid peroxidation and cause various biofilm damage, mitochondrial and lysosomal dysfunction, and cytotoxic effects $[19,20]$. Too much iron can also be deposited in human tissues, causing a condition called hemochromatosis [21]. Studies have linked iron overload to hepatic disease [22], heart disease [23], diabetes [24], and leukemia [25].
On top of that, iron carries a very high risk of cancer [26]. The enhancement of lipid peroxidation provoked by excessive iron leads to the imbalance of oxidative and antioxidant systems in the body, which directly damages DNA and induces gene mutations, leading to the activation of proto-oncogenes and the inactivation of tumor suppressor genes [27]. It has been reported that iron metabolism plays a complex role in forming and developing tumors at various stages and iron overload is correlated with tumors of the liver [28], colon [29], lung [30], esophagus [31], bladder [32], and other organs. After the tumor's formation, cancer cells become "addicted" to iron [33]. Cancer cells are characterized by vigorous division, so the synthesis and accumulation of many enzymes related to DNA synthesis, replication, and repair are needed in a short division cycle. Iron is a rate-limiting factor in DNA synthesis due to its role as an active center component in several DNA polymerases as well as repair enzymes related to chromosome stability $[11,12]$. Accordingly, iron is closely related to tumor angiogenesis and tumor cell invasiveness and is extremely valuable for tumors.

Notably, iron has a bidirectional effect in tumors: iron promotes tumor growth in the early stage, and sparks ferroptosis when excessive [34]. Ferroptosis is a process of cell death driven by cell metabolism and irondependent lipid peroxidation $[35,36]$. More and more evidences show that ferroptosis may participate in the genesis and development of tumors, promoting tumor cells' death by activating multiple regulatory sites of its pathway, and plays a vital role in tumor inhibition [35, 37, 38].

\section{Application of iron-based nanomaterials in the diagnosis and treatment of tumors}

\subsection{Superparamagnetic iron oxide nanoparticles in magnetic nanoparticles}

Here are many sorts of magnetic nanoparticles (MNPs), such as iron oxides, mixed oxides of iron and excessive metals, and MNP of iron and its alloys [40]. Magnetite $\left(\mathrm{Fe}_{3} \mathrm{O}_{4}\right)$ and gamma-hematite $\left(\gamma-\mathrm{Fe}_{2} \mathrm{O}_{3}\right)$ NPs, the prominent representatives of MNPs, are easy to synthesize and have good biocompatibility biodegradation [41, 42]. Also, both iron oxides contain atomic vacancies and polar amphoteric $\mathrm{OH}$ modifications and are therefore easily manipulated and functionalized by the surface [41]. These advantages make them widely concerned in the field of medicine and pharmaceutical. Because they are superparamagnetic, they are often referred to as SPIONs.

Above all, early diagnosis and localization of tumor is the key to tumor treatment. SPION as an MRI contrast agent can significantly enhance magnetic resonance signal intensity; imaging contrast and sensitivity are very high. This phenomenon can be ascribed to the contrast agent made from SPION has a particular distribution in 
reticuloendothelial cell-rich organs such as the liver, spleen, lymph, and bone marrow [43].

For oncology, SPION has an essential drug delivery function. Involving drugs into SPION can improve drug targeting, increase drug dose to cancerous tissues, and restrict drug side effects. The SPION coating provides an anchor point at which the drug molecules can be coupled. Significantly, SPION's surface can be modified explicitly by peptides, antibodies, and small molecules to recognize tumor-specific markers, tumor angiogenesis markers, and other tumor characterization-related substances, which is of magnificent weight to heighten the targeting to the affected areas [44-46].

Hollow SPION is utilized for drug loading and holds a high drug loading capacity is beyond doubt [47]. Currently, several drugs have been used in combination with SPION for cancer chemotherapy, including doxorubicin (DOX) [48], paclitaxel [49], methotrexate [50], and others.

Along with chemotherapy drugs, SPION can also be used as a vector for gene therapy. Gene therapy is a kind of anticancer technology by introducing exogenous genetic material into cancer cells through the carrier to set some specific proteins' expression to kill cancer cells [51]. One of the critical steps is gene delivery. Researches have revealed that the conjugation of bioactive molecules such as siRNA, oligos, and genes with SPION augments the intracellular pharmacokinetics and cell membrane penetration of nucleic acids [42, 52].

It is worth noting that targeted drug delivery across the blood-brain barrier (BBB) has always been a challenging technology, and MNP-based drug carriers hold promise for the imaging and treatment of primary and metastatic brain tumors. Several recent studies have reported using an external magnetic field to guide SPION to penetrate BBB in the treatment of glioma, the most common primary intracranial tumor [41, 53].

The superparamagnetism of SPION is a very serviceable property. In the external magnetic field, besides guiding drug transportation and enrichment, heat can also be generated in the target area to locally ablates the tumor cells. This therapy is called magnetic fluid hyperthermia (MFH) [54, 55].

MFH has many advantages. First, the thermal activation of SPION selectively damages cancer cells over a specific temperature range, for tumors are more susceptible to heat than normal tissue [56]. Additionally, MFH can also improve cancer cells' high sensitivity to chemotherapy by improving the permeability of the cancer cell membrane and heighten the absorption rate of anti-cancer drugs [56, 57]. Furthermore, SPION has a good penetration to targets deep in the biological system, and the presence of a magnetic field does not affect living biological tissue. Hence, SPION based MFH is a promising non-invasive treatment method.

Not only that, but the magnetofluid's ability to generate heat is also of particular interest for a driver for biological/chemotherapeutic drug release [55, 58, 59]. Strategies that rely on environmental and molecular markers to trigger drug release are often bounded by heterogeneity and lack of selectivity within the target tissue. Exogenous strategies that count on light or ultrasound energy may affect adjacent tissues and cause damage. The use of magnetic nanoparticles linked with an external magnetic field to selectively guide the accumulation and release of drugs in target tissues can minimize the impact on surrounding tissues.

Last but not least, the combined therapeutic properties of SPION have been widely studied $[41,54]$. That is, the above systems of SPION can render the dual treatment of MFH and chemotherapy for tumors, or an integrated therapeutic effect, which has a significant advantage vied with the single drug administration system, illustrating that using a combination of imaging, targeting, and treatment of the innovative drug delivery vehicle is the most promising direction.

\subsection{Iron-based metal-organic frameworks}

MOFs, a kind of emerging hybrid porous materials, possess a periodic structure formed by coordination between metal ions and organic groups. They usually have regular porosity, large specific surface area, and high pore volume. Moreover, the adjustable size, composition, structure, high dose drug/enzyme loading, selective functionalization of surface chemistry, improved biocompatibility, and low cytotoxicity make them promising clinical oncology treatment platforms $[60,61]$. Considering toxicity, biocompatibility, and biodegradability, the various available iron-containing MOFs offered many possibilities for the clinical application of controlled release of pharmacological molecules and are currently the most widely studied drug delivery MOFs. Next, the application potential of ironbased MOFs will be focused on tumor diagnosis and treatment.

First of all, iron-containing MOFs have superior drug delivery capabilities and, like MNPs, possess capabilities of magnetic imaging and stimulus reactivity release.

Moreover, photodynamic therapy (PDT), photothermal therapy (PTT), and chemodynamic therapy (CDT) based on specific factors such as acidic $\mathrm{pH}$, endogenous $\mathrm{H}_{2} \mathrm{O}_{2}$, and low catalase expression, that are prevalent in the tumor microenvironment (TME), have made promising advancement in tumor therapy $[62,63]$. On the ground of superior drug loading rate and catalytic activity, iron-based MOFs show great synergistic antitumor potential when used in combination with them. These methods based on iron-containing MOFs and their merged use with each other are described in detail in the following sections.

The principle of PDT is to utilize non-toxic light, photosensitizer, and molecular oxygen to yield highly active singlet oxygen $\left({ }^{1} \mathrm{O}_{2}\right)$ to cause tumor cell death [64]. Traditional small molecule photosensitizers have the disadvantages of poor water solubility and low light penetration depth [65]. In addition, the therapeutic efficacy of PDT was severely impaired by TME hypoxia [66]. Iron-based MOF displays extraordinary potential in PDT for the following advantages [67]: 1.) The regular crystal structure makes the photosensitizer molecules wrapped separately to avoid photosensitizer aggregation quenching. 2.) The pore structure is conducive to the 
diffusion of singlet oxygen. 3.) Superior biocompatibility. 4.) Excellent water solubility and biological enrichment function endowed by the nanometer-size effect. 5.) Through the catalysis of the released iron, the hypoxia of TME can be improved, raising the PDT efficiency. Wang et al. [68] established an intelligent multifunctional diagnostic and therapeutic DDS based on Fe-MOF, loaded with the chemotherapeutic drug dihydroartemisinin and the photosensitizer methylene blue, in conjunction with chemotherapy-photodynamic therapy. The release of Fe ions from MOFs in the tumor environment intensified the efficacy of DHA and catalyzed the release of $\mathrm{H}_{2} \mathrm{O}_{2}$ from $\mathrm{O}_{2}$, which further augmented the force of PDT.

Also induced by a laser, PTT utilizes photothermal conversion to ablate cancer cells $[63,69]$. In recent years, PTT is progressively attractive because of its noninvasive, non-toxic, targeting property and other advantages [70]. Zhu et al. [71] synthesized a Fe-DOX bio-MOF adsorbing the photosensitizer indocyanine green (ICG) through a step-by-step assembly strategy. The loading of ICG enables the NPs to have excellent photothermal conversion capability, triumphantly introducing PTT and PDT into one platform. The thermal effect provoked by PTT can accelerate the blood circulation in the tumor site and improve vascular permeability, thus promoting the enrichment and endocytosis of chemotherapy drugs. Reactive oxygen species (ROS) produced by PDT can effectively damage tumor cells in near-infrared (NIR) treated areas. Moreover, the iron-containing MOF shell structure can also be adopted as a T2-dominated contrast agent, which is expected to be used in real-time monitoring MR/PA/PT imaging. Therefore, this MOF provides a new idea for realizing multimodal imaging and the combination therapy of $\mathrm{PT} / \mathrm{PD} /$ chemotherapy.

To date, most multimodal therapies like PDT/ chemotherapy, PDT/PTT, and PDT/PTT/ chemotherapy have been based primarily on exogenous physical irradiation. Unfortunately, these exogenous radiations may damage healthy tissue adjacent to the tumor and have limited penetration into deep tumor tissue $[65,66]$. In 2016, the Bowen $\mathrm{Bu}$ team proposed a new kind of tumor treatment strategy: chemodynamic therapy (CDT). This therapy takes advantage of the weak acid microenvironment of the tumor as the reaction conditions, overexpressed $\mathrm{H}_{2} \mathrm{O}_{2}$ as reaction raw materials, and transition metal nanomaterials as a catalyst, by triggering Fenton reaction, catalyzing $\mathrm{H}_{2} \mathrm{O}_{2}$ to produce hydroxyl radicals $(\cdot \mathrm{OH})$ and other kinds of ROS, oxidizing tumor cell membrane, protein, and DNA molecules, eventually inducing tumor cell apoptosis [72]. Through the endogenous biochemical cascade induced by TME, CDT can solve the deep penetration problem without phototoxicity and has the inherent TME-specific targeting property [67], thus becoming an innovative anticancer strategy.

Though, CDT also has disadvantages. For instance, the weakly acidic $\mathrm{pH}$ and $\mathrm{H}_{2} \mathrm{O}_{2}$ concentration in TME are minimal for effective CDT [73]. One of the powerful methods to promote CDT efficiency is introducing more metal ions into tumor tissue to catalyze the Fenton reaction. The unique structure of MOFs enables them to decompose and release metal ions in the TME. Iron ion is the most effective catalyst for the Fenton reaction. Therefore, Fe-based MOF after the TME's degradation can be accepted as an effective inducer of the Fenton reaction [74, 75]. For example, the team research of Professor Sun [74] reported a MOF based on iron (III) carboxylate (HG-MIL@PDANPs). The presence of PDANPs enables it to achieve NIR-controlled sitespecific PTT. Besides, the core-shell NPs enhanced the release of $\mathrm{Fe} 3+$ and Gox through pH-triggered and NIR stimulation. The Fenton reaction driven by $\mathrm{Fe} 2+$ and the glutathione (GSH) consumption by $\mathrm{Fe} 3+$ contributed to $\cdot \mathrm{OH}$ production for CDT. The acidity recovery and $\mathrm{H}_{2} \mathrm{O}_{2}$ self-sufficiency catalyzed by GOX greatly promoted the reaction. Therefore, it is a comprehensive nanoplatform for tumor-specific PTT/CDT synergistic therapy, providing a new pathway for tumor-specific therapy.

Also, the Fenton reaction can induce the aggregation of lipid peroxides (LPO), resulting in ferroptosis. Ironcontaining MOF nano-enzymes holding ferroptosisrelated substances such as erastin [76], sorafenib [77], and P53 [78] plasmid could synergically entail ferroptosis through increasing LPO with the release of iron ions and ferroptosis inducers. Recently, Liu et al. [77] studied Fe-MOF carrying sorafenib for the treatment of liver cancer. The system can consume GSH, reduce GPX-4 levels, and enhance LPO production while offering iron ions to hasten ferroptosis induction, having significant therapeutic advantages.

In conclusion, these iron-containing MOF nanoenzymes can simulate the action of natural enzymes such as catalase. By causing biocatalytic cascade, they can improve the therapeutic effect of tumors in several aspects, such as improving tumor hypoxia, enhancing ROS damage by Fenton reaction and activating ferroptosis pathway. Many scientists are working to develop methods to prepare MOFs with specific morphology of mesoporous or macroporous particles, improve the loading and release capacity of guest molecules, and gradually improve the degradation mechanism, drug delivery kinetics, and toxicity studies of MOF

\section{Nanomaterials based on the development of important molecules in iron homeostasis metabolism for tumor applications}

A large proportion of DDS have defects specific to the type of DDS, including lack of biocompatibility and biodegradation, low stability, structural heterogeneity, undesirability immune response and antigenicity, and cytotoxicity [5]. These shortcomings can be overwhelmed by applying protein-based DDS from target organisms. In general, they have the advantages of high histocompatibility, easy absorption, good metabolism, and low toxicity. As mentioned earlier, cancer cells are iron-eating on account of their demand for the energy to proliferate rapidly. As a transmembrane protein that regulates iron metabolism, TFR1 is 
expressed at much higher levels in various cancer cells than in normal cells [13, 79]. More importantly, it possesses two inherently potent endogenous binding ligands: TF and Ft. Therefore, this receptor is a potential target of the DDS in anti-tumor therapy.

\subsection{Nanoparticles targeting transferrin receptors}

A highly compatible complex is formed after the interaction between TFR1 on the cell surface with TF, then internalized by the cell membrane through receptormediated endocytosis. In this context, NPs carrying TF may lead to more selective cell uptake and enhanced therapeutic efficacy. A TF functionalized porous silicon nanoparticle (Tf@pSiNPs ) was investigated to target the DOX to glioma cells across the BBB [79]. It was demonstrated in vitro that TF functionalization improved pSiNP vector internalization efficiency through clathrinmediated endocytic mechanisms. Therefore, TF functionalization can update the vector's targeting, improve the therapeutic effect, and lessen drug-related side effects.

However, with high endogenous TF concentrations in the blood, TF-modified nanocarriers may exhibit less than desirable targeting capabilities and safety [80]. Ft, another natural ligand for TFR1, consists of a spherical nanocage protein shell and an iron core that regulates iron storage [5]. The apoferritin shell can be reversibly assembled/decomposed at different $\mathrm{pH}$ values to encapsulate the selected drug into a cavity. This process can provide biocompatible DDS, allowing repetitive production and many surface modification options.

Conversion of $\mathrm{Ft}$ drug carriers in vivo is often impeded by the drug loading process's inefficiency and low yield [81]. Xiyun Yan [82] and his team found that natural drug entry channels in the shell of recombinant human $\mathrm{H}-\mathrm{Ft}$ (HFn) are susceptible to temperature changes. Based on this, a simple drug delivery strategy counted on channels was developed to avoid the denaturation of HFn protein cage and denaturant. Experimental results revealed that the drug loading rate of DOX was significantly higher than that of the denaturation-based method. Moreover, the HFn-DOX produced by the channel-based loading strategy had better stability, higher biosafety, and enhanced antitumor activity. Besides, the Ft drug channel is universal for small molecule drug loading, so it can be developed into a new drug technology platform.

\section{Conclusions}

Iron possesses excellent physical properties such as electricity, magnetism, and heat. The regulation network of iron metabolism is elaborate and complex, and is closely related to many kinds of diseases, especially tumors. These characteristics of iron can be employed in the field of tumor diagnosis and treatment based on nanomaterials. Its main functions for tumors include 1 . Under the action of external magnetic field, iron's magnetic properties can be used to diagnose tumors by MRI, target tumors to aggregate drugs, "cook" tumor cells by magneto-thermal conversion, and control thermal-touch drug release [2]. Iron can improve TME hypoxia by catalyzing the Fenton reaction, assisting the efficacy of PDT, PTT, and CDT, causing more severe oxidative damage to tumor cells; moreover, the Fenton reaction can also cause the accumulation of LPO, thus inducing ferroptosis [3]. Targeting the overexpressed transferrin receptor of tumor cells and using its affinity with transferrin/ferritin to enhance the targeting of nanoparticles [4]. Own to the critical role of iron in the human body, iron-related NPs usually have low toxicity, degradability, and good biocompatibility. Consequently, iron-based nanoparticles and nanomaterials based on important molecules of iron homeostasis-metabolism show great potential in the field of tumor diagnosis and treatment.

With the deepening of iron metabolism research, the complex molecular mechanism of iron in vivo, the physiological significance of iron in various tissues and organs, and the role of iron in various tumor models will be gradually revealed. All those will helpe people continue to explore the application value of iron in the field of cancer and then create more and more valuable iron-related nanomaterials.

\section{References}

1. S.K. Gulla, R. Kotcherlakota, S. Nimushakavi, V. Nimmu, S. Khalid, C.R. Patra, A. Chaudhuri, ACS Omega 3 (2018), 8663-8676.

2. D. Wu, Y. Chen, S. Wen, Y. Wen, R. Wang, Q. Zhang, G. Qin, H. Yi, M. Wu, L. Lu, X. Tao, X. Deng, Nanoscale Res. Lett. 14 (2019).

3. M. Yang, J. Li, P. Gu, X. Fan, Bioact. Mater. 6 (2021), 1973-1987.

4. M.W. Hentze, M.U. Muckenthaler, N.C. Andrews, Cell 117 (2004), 285-297.

5. Z. Skubalova, S. Rex, M. Sukupova, M. Zahalka, P. Skladal, J. Pribyl, H. Michalkova, A. Weerasekera, V. Adam, Z. Heger, Int. J. Nanomed. 16 (2021), 114.

6. M.A. Fernandes, J.O. Eloy, M.T Luiz, L.R..J. Sergio, C.B. Júlio, L.R. de la Fuente, C.O.-de S. Luis, J. M. Marchetti, M.J. Santos-Martinez, M. Chorilli, Colloids Surf., 611 (2021).

7. S. Balesaria, R. Hanif, M.F. Salama, K. Raja, H.K. Bayele, H. McArdle, S.K. Srai, Haematologica 97 (2012), 661-669.

8. B. Chen, G.F. Li, Y. Shen, X.I. Huang, Y.J. Xu, Exp. Ther. Med. 10 (2015), 7-11.

9. H. Wu, H. Wei, D. Zhang, S.A. Sehgal, D. Zhang, X. Wang, Y. Qin, L. Liu, Q. Chen, Redox Biol. 36 (2020), 101661.

10. A. Gabizon, M. Bradbury, U. Prabhakar, W. Zamboni, S. Libutti, P. Grodzinski, Lancet 384 (2014), 2175-2176.

11. E.G. Farrow, X. Yu, L.J. Summers, S.I. Davis, J.C. Fleet, M.R. Allen, A.G. Robling, K.R. Stayrook, V. Jideonwo, M. J. Magers, H. J. Garringer, R. Vidal, 
R.J. Chan, C.B. Goodwin, S.L. Hui, M. Peacock, K.E. White, PNAS 108 (2011), 18587-18588.

12. D.J. Netz, C.M. Stith, M. Stumpfig, G. Kopf, D. Vogel, H.M. Genau, J.L. Stodola, R. Lill, P.M. Burgers, A.J. Pierik, Nat. Chem. Biol. 8 (2011), 125-132.

13. H. Choudhury, M. Pandey, P.X. Chin, Y.L. Phang, J.Y. Cheah, S.C. Ooi, Mak K.K., M.R. Pichika, P. Kesharwani, Z. Hussain, B. Gorain, Drug Delivery Transl. Res. 8 (2018), 1545-1563.

14. Y. Beguin, M. Aapro, H. Ludwig, L. Mizzen, A. Osterborg, Crit. Rev. Oncol. Hematol. 89 (2014), 115 .

15. M.W. Hentze, M.U. Muckenthaler, Andrews N.C., Cell 117 (2004), 285-297.

16. T. Ganz, Blood 117 (2011), 4425-4433.

17. S. Keshav, R. Stevens, Br. J. Gen. Pract. 67 (2017), 10-11.

18. M. Tang, D.N. Frank, A.E. Hendricks, Ir D., F. Esamai, E. Liechty, K.M. Hambidge, Krebs N.F., Nutrients 9 (2017).

19. D.L. Zhang, M.C. Ghosh, T.A. Rouault, Front. Pharmacol. 5 (2014).

20. R. Gozzelino, P. Arosio, Front. Pharmacol. 6 (2015).

21. M. Dostalikova-Cimburova, K. Kratka, K. Balusikova, J. Chmelikova, V. Hejda, J. Hnanicek, J. Neubauerova, J. Vranova, J. Kovar, J. Horak, J. Cell. Mol. Med. 16 (2012), 1816-1826.

22. P. Zhabyeyev, S.K. Das, R. Basu, M. Shen, V.B. Patel, Z. Kassiri, G.Y. Oudit, Am. J. Physiol. Heart Circ. Physiol. 314 (2018), H978-H990.

23. H. Nam, C.Y. Wang, L. Zhang, W. Zhang, S. Hojyo, T. Fukada, M.D. Knutson, Haematologica 98 (2013), 1049-1057.

24. S.V. Shah, V.A. Fonseca, Diabetes Care 34 (2011), 1676-1677.

25. N.E. Hellman, M. Schaefer, S. Gehrke, P. Stegen, W.J. HoVman, J.D. Gitlin, W. Stremmel, Gut 47 (2000), 858-860.

26. S.J. Dixon, B.R. Stockwell, Nat. Chem. Biol. 10 (2014), 9-17.

27. C.C. Xue, M.H. Li, Y. Zhao, J. Zhou, Y. Hu, K.Y. Cai, Y. Zhao, S.H. Yu, Luo Z., Sci. Adv. 6 (2020).

28. C. Wang, J.L. Babitt, Blood 133 (2019), 18-29.

29. X. Xue, S.K. Ramakrishnan, K. Weisz, D. Triner, ie L., D. Attili, A. Pant, B. Gyorffy, M. Zhan, C. Carter-Su, K.M. Hardiman, T.D. Wang, M.K. Dame, J. Varani, D. Brenner, E.R. Fearon, Y.M. Shah, Cell Metab. 24 (2016), 447-461.

30. S.M. Cloonan, S. I.M. Mumby, A.M.K. Choi, K.F. Chung, G.J. Quinlan, Am. J. Respir. Crit. Care Med. 196 (2017), 1103-1112.

31. S.J. Ford, P. Obeidy, D.B. Lovejoy, M. Bedford, L. Nichols, C. Chadwick, O. Tucker, G.Y. Lui, D.S. Kalinowski, P.J. Jansson, T.H. Iqbal, D. Alderson,
D.R. Richardson, C. Tselepis, Br. J. Pharmacol. 168 (2013), 1316-1328.

32. N. Dikshit, P. Bist, S.N. Fenlon, N.K. Pulloor, C.E. Chua, M.A. Scidmore, J.A. Carlyon, B.L. Tang, S.L. Chen, Sukumaran, B., 11 (2015).

33. D. Basuli, L. Tesfay, Z. Deng, B. Paul, Y. Yamamoto, G. Ning, W. Xian, F. McKeon, M. Lynch, C.P. Crum, P. Hegde, M. Brewer, X. Wang, L.D. Miller, N. Dyment, F.M. Torti, S.V. Torti, Oncogene 36 (2017), 4089-4099.

34. I. Ingold, M. Conrad, Oncotarget 9 (2018), 22241 22242.

35. Yi J., A.M. Minikes, X. Jiang, Biol. 26 (2019), 621622.

36. B.R. Stockwell, X. Jiang, Cell Metab 30 (2019), 1415.

37. R. Kang, G. Kroemer, D. Tang, Free Radic. Biol. Med. 133 (2019), 162-168.

38. W. Wang, M. Green, J.E. Choi, M. Gijon, P.D. Kennedy, J.K. Johnson, P. Liao, X. Lang, I. Kryczek, A. Sell, H. Xia, J. Zhou, G. Li, J. Li, W Li, S. Wei, L. Vatan, H. Zhang, W. Szeliga, W. Gu, R. Liu, T.S. Lawrence, C. Lamb, Y. Tanno, M. Cieslik, E. Stone, G. Georgiou, T.A. Chan, A. Chinnaiyan, W. Zou, Nature 569 (2019), 270-274.

39. B. Yu, B. Choi, W. Li, D.H. Kim, Nat. Commun. 11 (2020).

40. H. Zhang, L. Li, X.L. Liu, J. Jiao, C.T. Ng, J.B. Yi, Y.E. Luo, B.H. Bay, L.Y. Zhao, M.L. Peng, N. Gu, H.M. Fan, ACS Nano 11 (2017), 3614-3631.

41. L.L. Israel, A. Galstyan, E. Holler, J.Y. Ljubimova, J. Controlled Release 320 (2020), 45-62.

42. J. Pan, W. Ruan, M. Qin, Y. Long, T. Wan, K. Yu, Y. Zhai, C. Wu, Y. Xu, Sci. Rep. 8 (2018).

43. P. Wang, W. Sun, J. Guo, K. Zhang, Y. Liu, Q. Jiang, D. Su, X. Sun, Colloids Surf., 197 (2021), 111403.

44. S. Zhang, M. Gong, D. Zhang, H. Yang, F. Gao, L. Zou, Eur. J. Radiol. 83 (2014), 1190-1198.

45. M.A. Shevtsov, L.Y. Yakovleva, B.P. Nikolaev, Y.Y. Marchenko, A.V. Dobrodumov, K.V. Onokhin, Y.S. Onokhina, S.A. Selkov, A.L. Mikhrina, I.V. Guzhova, M.G. Martynova, O.A. Bystrova, A.M. Ischenko, B.A. Margulis, Neuro-Oncol. 16 (2014), 38-49.

46. C. Ansari, G.A. Tikhomirov, S.H. Hong, R.A. Falconer, P.M. Loadman, J.H. Gill, R. Castaneda, F.K. Hazard, L. Tong, O.D. Lenkov, D.W. Felsher, J. Rao, H.E. Daldrup-Link, Small 10 (2014), 566-575.

47. K. Cheng, S. Peng, C. Xu, S. Sun, JACS 131 (2009), 10637-10644.

48. C. Sun, O. Veiseh, J. Gunn, C. Fang, S. Hansen, D. Lee, R. Sze, R.G. Ellenbogen, J. Olson, M. Zhang, Small 4 (2008), 372-379.

49. A. Sato, N. Itcho, H. Ishiguro, D. Okamoto, N. Kobayashi, K. Kawai, H. Kasai, D. Kurioka, H. 
Uemura, Y. Kubota, M. Watanabe, Int. J. Nanomed. 8 (2013), 3151-3160.

50. N. Kohler, C. Sun, A. Fichtenholtz, J. Gunn, C. Fang, M. Zhang, Small 2 (2006) 785-792.

51. R. Ortiz, C. Melguizo, J. Prados, P.J. Álvarez, O. Caba, F. Rodríguez-Serrano, F. Hita, A. Aránega, Anti-Cancer Drug Discovery 7 (2012), 297-312.

52. A. Sagner, Z.B. Gaber, J. Delile, J.H. Kong, D.L. Rousso, C.A. Pearson, S.E. Weicksel, M. Melchionda, S.N. Mousavy Gharavy, J. Briscoe, B.G. Novitch, PLoS Biol. 16 (2018).

53. M.A. Shevtsov, B.P. Nikolaev, L.Y. Yakovleva, Y.Y. Marchenko, A.V. Dobrodumov, A.L. Mikhrina, M.G. Martynova, O.A. Bystrova, I.V. Yakovenko, A.M. Ischenko, Int. J. Nanomed. 9 (2014), 273-287.

54. S.P.C. Hsu, U. Dhawan, Y. Tseng, C. Lin, C. Kuo, L. Wang, R. Chung, Appl. Mater. Today 18 (2020).

55. R.R. Shah, A.R. Dombrowsky, A.L. Paulson, M.P. Johnson, D.E. Nikles, C.S. Brazel, Sci. Eng. 68 (2016), 18-29.

56. R.D. Piazza, W.R. Viali, C.C. dos Santos, E.S. Nunes, R.F.C. Marques, P.C. Morais, S.W. da Silva, J.A.H. Coaquira, M. Jafelicci, Mater. Res. Express 7 (2020).

57. N. Orsolic, D. Odeh, M.J. Jembrek, J. Knezevic, D. Kucan, Molecules 25 (2020), 3271.

58. J.F Liu., B. Jang, D. Issadore, A. Tsourkas, Rev.: Nanomed. Nanobiotechnol. 11 (2019).

59. J.F. Liu, N. Neel, P. Dang, M. Lamb, J. McKenna, L. Rodgers, B. Litt, Z. Cheng, A. Tsourkas, D. Issadore, Small 14 (2018).

60. M.X. Wu, Y.W. Yang, Adv. Mater. 29 (2017).

61. G. Tan, Y. Zhong, L. Yang, Y. Jiang, J. Liu, F. Ren, Chem. Eng. J. 390 (2020).

62. Y. Han, S. Gao, Y. Zhang, Q. Ni, Z. Li, X.J. Liang, J. Zhang, Bioconjug. Chem. 31 (2020), 1247-1258.

63. K. Zhang, X. Meng, Y. Cao, Z. Yang, H. Dong, Y. Zhang, H. Lu, Z. Shi, X. Zhang, Adv. Funct. Mater. 28 (2018).

64. X. Lu, S. Gao, H. Lin, J. Shi, Small (2021).

65. P.T. Wu, C.L. Lin, C.W. Lin, N.C. Chang, W.B. Tsai, J. Yu, Nanomaterials 9 (2018).

66. K. Zhang, Y. Zhang, X. Meng, H. Lu, H. Chang, H. Dong, X. Zhang, Biomaterials 185 (2018), 301-309.

67. A. Bieniek, A.P. Terzyk, M. Wiśniewski, K. Roszek, P. Kowalczyk, L. Sarkisov, S. Keskin, K. Kaneko, Prog. Mater. Sci. (2020).

68. C. Wang, X. Jia, W. Zhen, M. Zhang, X. Jiang, ACS Biomater. Sci. Eng. 5 (2019), 4435-4441.

69. Z. Lv, J. Li, F. Yang, K. Cao, Q. Bao, Y. Sun, J. Yuan, Front. Bioeng. Biotechnol. 8 (2020).

70. B. Zhao, H. Wang, W. Dong, S. Cheng, H. Li, J. Tan, J. Zhou, W. He, L. Li, J. Zhang, G. Luo, W.J. Qian, Nanobiotechnol. 18 (2020).
71. Y. Zhu, N. Xin, Z. Qiao, S. Chen, L. Zeng, Y. Zhang, D. Wei, J. Sun, H. Fan, Adv. Healthc. Mater. 9 (2020).

72. L. He, Q. Ni, J. Mu, W. Fan, L. Liu, Z. Wang, L. Li, W. Tang, Y. Liu, Y. Cheng, L. Tang, Z. Yang, Y. Liu, J. Zou, W. Yang, O. Jacobson, F. Zhang, P. Huang, X. Chen, JACS 142 (2020), 6822-6832.

73. X. Liu, Y. Jin, T. Liu, S. Yang, M. Zhou, W. Wang, H. Yu, ACS Biomater., Sci. Eng. 6 (2020), 48344845.

74. H. Wu, D. Gu, S. Xia, F. Chen, C. You, B. Sun, Biomater. Sci. 9 (2021), 1020-1033.

75. H. Ranji-Burachaloo, F. Karimi, K. Xie, Q. Fu, P.A. Gurr, D.E. Dunstan, G.G. Qiao, ACS Appl. Mater. Interfaces. 9 (2017), 33599-33608.

76. H. Xin, F. Wang, R. Luo, Lei J., Chemistry 27 (2021), 4307-4311.

77. X. Liu, X. Zhu, X. Qi, X. Meng, K. Xu, Int. J. Nanomed. 16 (2021), 1037-1050.

78. C. Zhang, W. Bu, D. Ni, S. Zhang, Q. Li, Z. Yao, J. Zhang, H. Yao, Z. Wang, J. Shi, Angew. Chem. Int. Ed. Engl. 55 (2016), 2101-2106.

79. M. Luo, G. Lewik, J.C. Ratcliffe, C.H.J. Choi, E. Makila, W.Y. Tong, N.H. Voelcker, ACS Appl. Mater. Interfaces. 11 (2019), 33637-33649.

80. Y.C. Chen, C.F. Chiang, L.F. Chen, P.C. Liang, W.Y. Hsieh, W.L. Lin, Biomaterials 35 (2014), 4066-4081.

81. C. Pozzi, F. Di Pisa, C. Bernacchioni, S. Ciambellotti, P. Turano, S. Mangani, Acta Crystallogr. 71 (2015), 1909-1920.

82. B. Jiang, X. Chen, G. Sun, X. Chen, Y. Yin, Y. Jin, Q. Mi, L. Ma, Y. Yang, X. Yan, K. Fan, Nano Today 35 (2020). 